\title{
Complete Response to Dual Immunotherapy in a Young Adult with Metastatic Alveolar Soft Part Sarcoma Enabled by a Drug Recovery Program in a Community Practice
}

\author{
Alexandra Conry, BS, ${ }^{1}$ Melissa Peters, MD, ${ }^{2}$ Daniel B. Fried, MD, PhD, ${ }^{2}$ Amy Adams, RN, BSN, ${ }^{1}$ \\ Alfred W. Campbell, MD, ${ }^{2}$ James D. Bearden, MD, ${ }^{2}$ Robert D. Siegel, MD, ${ }^{1}$ and Howland E. Crosswell, MD ${ }^{1}$
}

\begin{abstract}
Alveolar soft part sarcoma (ASPS) is an extremely rare tumor that frequently occurs in adolescent and young adults (AYA). Survival is poor for patients with metastatic and/or relapsed disease not amenable to local control, and limited therapeutic options are available. A major barrier to cancer care in the United States AYA population is lack of access to coordinated care and appropriate therapies for those who lack insurance or who are underinsured. We report a 25-year-old unemployed, uninsured, single mother who presented with a $12.8 \times 21 \mathrm{~cm}$ soft tissue thigh mass with heterogeneous avidity, max standardized uptake value of 9 , with metastatic disease to the ipsilateral inguinal lymph nodes and to the bilateral lungs. After local control of the primary mass was obtained, a recently developed, comprehensive drug replacement program (DRP) was used to gain access to nivolumab, and after frank progression was noted, ipilimumab was added every 6 weeks. No biomarkers associated with response to immunotherapy were identified. After four cycles, a complete response was observed and patient remains disease free 36 months after beginning dual immunotherapy treatment. We obtained immunotherapy agents through a DRP and describe the development and the utility of this program in the community setting. Our report highlights both first documented sustained complete response to sequenced immunotherapy in an AYA with ASPS as well as a comprehensive DRP, which enabled access to therapy for our patient.
\end{abstract}

Keywords: adolescent young adult, alveolar soft part sarcoma, dual immunotherapy, financial assistance

\section{Background}

A LVEOLAR SOFT PART SARCOMA (ASPS) is an extremely rare tumor of mesenchymal origin, which accounts for $0.05 \%-1 \%$ of all soft tissue sarcoma (STS) or about 100 cases annually in the United States. ${ }^{1}$ It is characterized by a somatic translocation between chromosomes $\mathrm{X}$ and 17 , which results in an ASPSCR1-TFE3 fusion gene, and is associated with metastasis to lung, bone, and brain in $40 \%$ of cases. ${ }^{2}$ ASPS is generally considered not sensitive to cytotoxic chemotherapy, and despite responses to targeted agents, 5-year survival is poor in patients with metastatic disease leading many to consider trials of novel therapies, including immunotherapy. ${ }^{3}$

The peak incidence of ASPS occurs in adolescents and young adults (AYA), an age group which has recently been recognized to have significant health care disparities. Re- cently defined by the National Cancer Institute (NCI) as ages 15-39 years at the time of diagnosis, AYAs have been reported to have frequent delays in diagnosis, suffer fragmentation of care between pediatric and oncology centers, display more nonadherence to therapy than younger and older counterparts, have higher rates of underinsurance or lack of insurance, inability to travel to major centers, and/or reduced clinical trial enrollment. ${ }^{4-9}$ Specifically in the United States, AYAs who lack insurance coverage at diagnosis, or who have public insurance coverage, have been shown to have more advanced stage of disease at diagnosis, receive less guidelinedirected care, and have poorer outcomes than their insured counterparts. $^{10,11}$ To address AYA cancer care disparities, many centers are developing dedicated, collaborative care programs involving both pediatric and medical oncology disciplines, which are focused on addressing many of the

\footnotetext{
${ }^{1}$ Bon Secours St. Francis Health System, St. Francis Cancer Center, Greenville, South Carolina, USA.

${ }^{2}$ Spartanburg Regional Health System, Gibbs Cancer Center \& Research Institute, Spartanburg, South Carolina, USA.

Prior Report: Abstract form published online by the Journal of Clinical Oncology: Targeting tumors and financial toxicity in AYAs: exceptional response to dual immunotherapy in an uninsured young adult with soft tissue sarcoma. J Clin Oncol. 2018;36(Suppl):abstract e23530.
} 
issues faced by AYAs. ${ }^{12-15}$ We describe a comprehensive drug replacement program (DRP), which was used to access dual immunotherapy for an underinsured AYA. Furthermore, we suggest that comprehensive DRPs similar to ours may be able to address significant issues of financial toxicity and access to care that AYAs frequently face and should be considered vital for United States-based AYA programs.

\section{Case Presentation}

A 25-year-old female presented with a 6-year history of increasing thigh pain and swelling. The patient was a single mother of two children, was unemployed and had no insurance at the time of referral to the regional AYA Program. Eastern Cooperative Oncology Group performance status was 3.

Magnetic resonance imaging (MRI) and computed tomography (CT) were performed and a positron emission tomography showed a $12.8 \times 21 \mathrm{~cm}$ soft tissue mass with heterogeneous avidity, max standardized uptake value of 9 , with metastatic disease to the ipsilateral inguinal lymph nodes and to the bilateral lungs (Fig. 1). Biopsy showed nests of epithelioid cells with eosinophilic cytoplasm and interdigitating vascular network, positive for periodic acid-Schiff, desmin, vimentin, and transcription factor E3 (TFE3). Nextgeneration sequencing showed ASPSCR1-TFE3 fusion, confirming the diagnosis of ASPS. Staging was pT2a (tumor

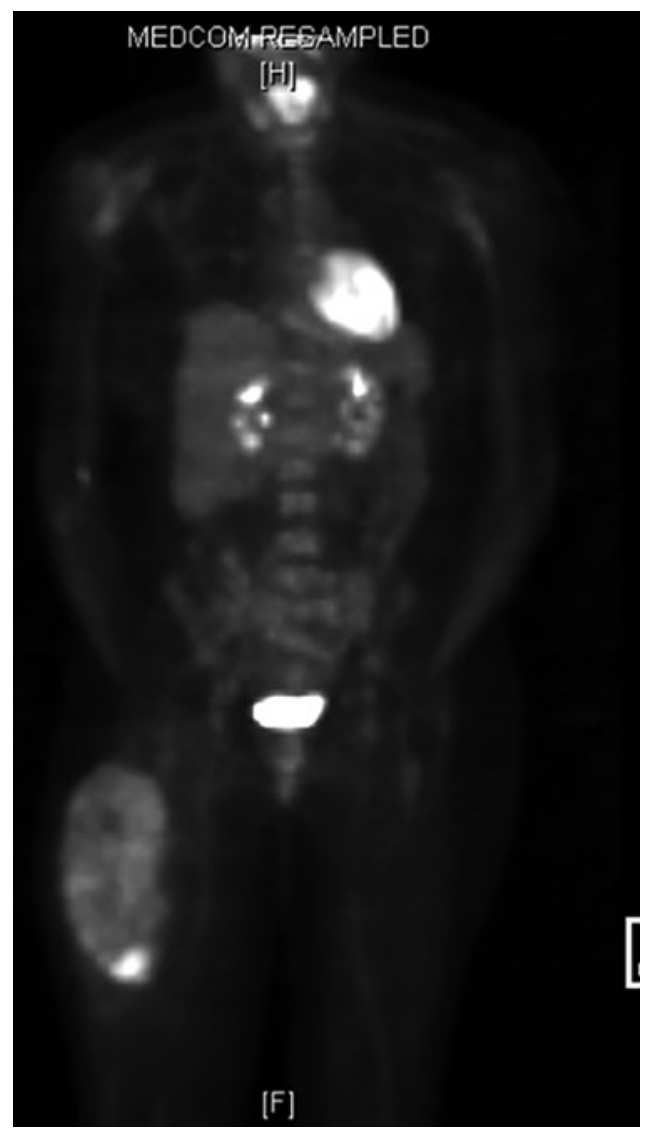

FIG. 1. Positron emission tomography demonstrating primary tumor in right proximal thigh and metastatic ipsilateral pelvic lymph nodes and bilateral pulmonary nodules. more than $5 \mathrm{~cm}$ in greatest dimension, superficial tumor), N1 (refers to the surrounding lymph nodes with cancer), M1 (cancer has spread to other parts of the body), stage IV disease.

Because of debilitating pain and lack of insurance, cytotoxic chemotherapy with doxorubicin and ifosfamide was attempted for one cycle, while radiation (RT) therapy to the primary site was planned and subsequently administered. Hospital sponsorship was rapidly obtained and a comprehensive DRP was used to gain access to pazopanib. The DRP was implemented the year before to reduce cost implications for our patients and lessen the financial impact of unreimbursed care on our institution, which is a significant issue among United States-based academic and community-based oncology programs. ${ }^{16,17}$ The DRP consists of 2.5 full-time employees to research opportunities for drug recovery for and provide financial counseling to the nearly 1700 patients cared for annually at our institution. DRP staff are trained to utilize available pharmaceutical foundations to replace inventory for those without insurance and in situations when a drug was delivered off-label without insurance authorization. ${ }^{18}$

Significant dose-limiting toxicities and nonadherence with further dose modifications limited response. ${ }^{18}$ After RT to the primary site, the patient had a complete resection of her thigh mass with negative margins and only $30 \%$ necrosis was noted. Worsening cough, dyspnea, and hypoxemia developed, and progressive disease in the lungs was noted at 2 and 6 months post initial presentation. Patient failed screening for three NCI-sponsored trials, which included two different immunotherapy trials and a precision medicine trial matching therapy to targeted exome sequence results (MATCH). Tissue analysis was negative for high tumor mutational burden, mismatch repair deficiency, and programmed death ligand 1 (PDL1) staining $(<1 \%)$. Federally sponsored insurance (Medicaid) was activated but denied attempts to treat the patient with immunotherapy despite signals of activity of immunotherapy in ASPS in the literature, ${ }^{3,19}$ DRP was again used to gain access to nivolumab, which was initiated at $240 \mathrm{mg}$ every 2 weeks. Progressive disease was noted with new pulmonary nodules after two cycles, and low-dose ipilimumab $1 \mathrm{mg} / \mathrm{kg}$ was added every 6 weeks, a regimen modeled after contemporary studies for rare tumors and other sarcomas. ${ }^{20}$ After two cycles of combination therapy, the patient had a near-complete response and at 6 months, the patient had no evidence of disease. Currently, the patient has no evidence of disease 36 months after initiation of dual immunotherapy started (Fig. 2).

\section{Discussion and Conclusions}

Standard therapies for localized ASPS include surgery and RT, and although multikinase inhibitors have single-agent activity, there are currently no standard therapies for metastatic ASPS and long-term survival remains less than $20 \% .^{21-23}$ The excellent activity and approval of immunotherapy in multiple adult solid tumors has led many to investigate both single agent and combinations of checkpoint inhibitors in rare tumors, including sarcomas. ${ }^{24-26}$ Recently, SARC028 trial, a multicenter phase II study of PD1 inhibitors in patients with sarcomas, reported a $19 \%$ response rate of pembrolizumab across four different STSs, none of which was ASPS. $^{26}$ The Alliance Cooperative group reported on 96 patients with STS who were randomly assigned to anti-PD1 

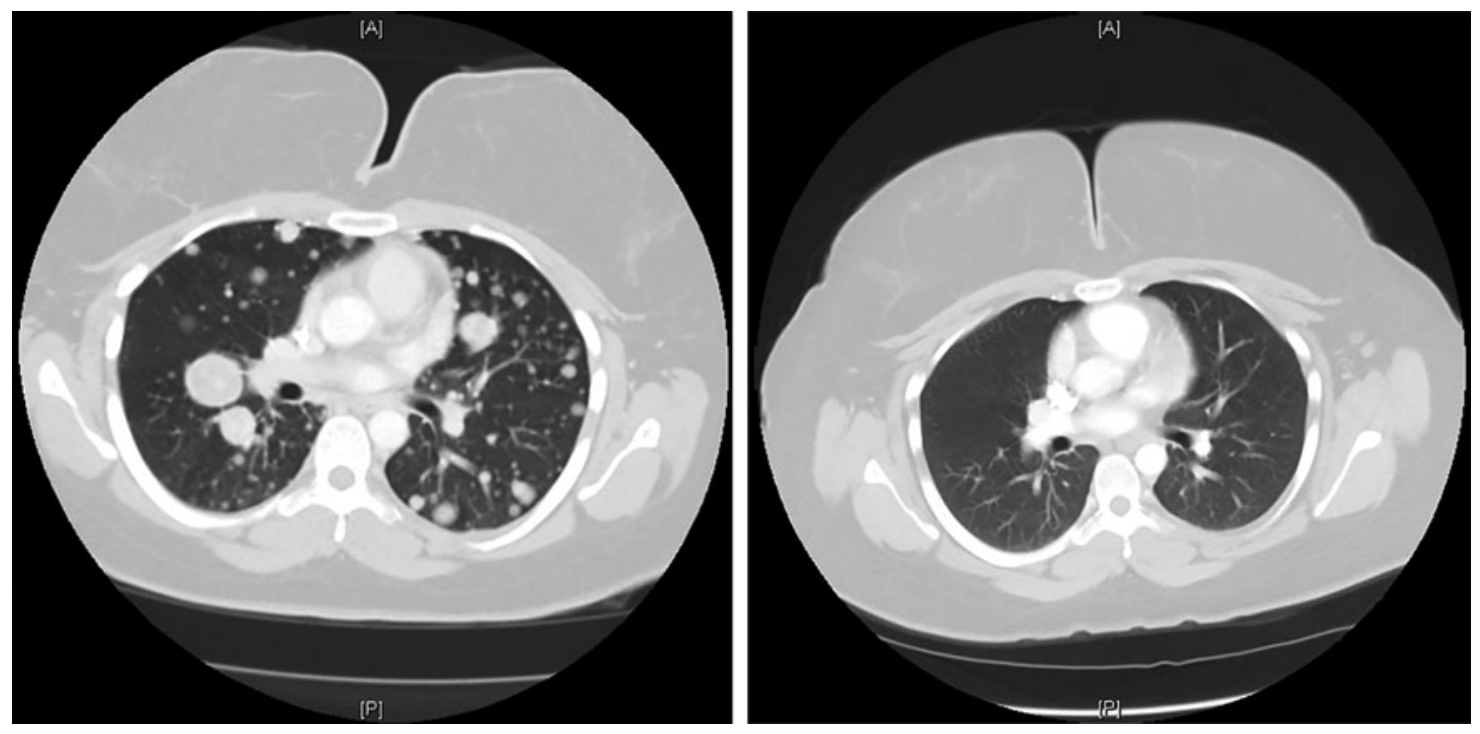

FIG. 2. Computed tomography before and after dual checkpoint inhibition demonstrating complete response.

with nivolumab alone or the combination of nivolumab and cytotoxic T-lymphocyte-associated protein 4 inhibitor ipilumumab and found a $5 \%$ and $16 \%$ response rate, respective$1 y .{ }^{20}$ Two of the 85 evaluable patients had ASPS, highlighting the rarity of the disease, and 1 patient with ASPS had a partial response to single-agent nivolumab. Preliminary data from a phase II study assessing PDL1 blockade using atezolizumab in ASPS were presented at the Connective Tissue Oncology Society annual meeting in 2018, where an objective response rate of $42 \%$ was seen in 19 patients with ASPS. ${ }^{27}$ Our patient's response was exceptional in that a sustained complete response has been maintained for 36 months on continued therapy with minimal toxicity. This case also highlights the significant impact that a comprehensive DRP can have on caring for under-resourced AYAs in United States communities.

The DRP at our institution consists of not only dedicated team of financial counselors, but also a pharmacist and AYA nursing and navigation that have established processes to quickly access hospital sponsorship and pharmaceutical sponsored-drug access programs to limit institutional costs and patient's financial toxicity. As compared with adult patients $>40$ years of age whose reasons for accessing the DRP were predominantly related to copay assistance for outpatient and oral medications, lack of insurance was the single most common reason for drug recovery in AYAs, accounting for $65 \%$ of the drugs recovered in the AYA population. ${ }^{18} \mathrm{We}$ consider this DRP to be integral to a broader AYA Program, which is designed to provide access and adherence to appropriate regimens, which are considered significant challenges for AYAs in the United States with no insurance. ${ }^{7,10,12-14}$ It is well recognized that AYAs require unique care paradigms and significant resources to address these and other issues. $7,12,14$

In conclusion, sequenced, dual immunotherapy resulted in a deep and durable complete remission in a young adult with metastatic ASPS and should be considered for further clinical study for patients with relapsed or metastatic ASPS. Additionally, dedicated care and coordinated financial assistance programs focused on underinsured AYAs may be considered essential components of specialized care of AYAs, particularly in the United States and other countries with hybrid public and private health care systems.

\section{Declarations}

Ethics approval and consent to participate: IRB approval was obtained from Bon Secours Richmond Health System (BSRHS) IRB, BSR \#CRS00000026 the Bon Secours St. Francis Adolescent Young Adult Study.

\section{Consent for Publication}

Consent for publication was obtained from the patient. A copy of the written consent is available for review.

\section{Author Contributions}

H.E.C., R.D.S. conceived and designed the study and case report; A.C., A.A., A.W.C., M.P., D.B.F., J.D.B., R.D.S., and H.E.C. contributed images and descriptions of case report and/or revision of the article; H.E.C., A.C. drafted the article and prepared the article for submission.

\section{Author Disclosure Statement}

No competing financial interests exist.

\section{Funding Information}

Patient sponsorship was obtained by the hospital from a comprehensive patient assistance program. Supported by St. Baldrick's Foundation Infrastructure Grant for Adolescent Young Cancer Research.

\section{References}

1. Folpe AL, Deyrup AT. Alveolar soft-part sarcoma: a review and update. J Clin Pathol. 2006;59(11):1127-32.

2. Orbach D, Brennan B, Casanova M, et al. Paediatric and adolescent alveolar soft part sarcoma: a joint series from 
European cooperative groups. Pediatr Blood Cancer. 2013; 60(11):1826-32.

3. Groisberg R, Hong DS, Behrang A, et al. Characteristics and outcomes of patients with advanced sarcoma enrolled in early phase immunotherapy trials. J Immunother Cancer. 2017;5(1):100.

4. Johnson RH, Macpherson CF, Smith AW, et al. Facilitating teamwork in adolescent and young adult oncology. J Oncol Pract. 2016;12(11):1067-74.

5. Keegan TH, DeRouen MC, Parsons HM, et al. Impact of treatment and insurance on socioeconomic disparities in survival after adolescent and young adult Hodgkin lymphoma: a population-based study. Cancer Epidemiol Biomarkers Prev. 2016;25(2):264-73.

6. Bleyer A, Morgan S, Barr R. Proceedings of a workshop: bridging the gap in care and addressing participation in clinical trials. Cancer. 2006;107(7 Suppl):1656-8.

7. Coccia PF, Pappo AS, Beaupin L, et al. Adolescent and young adult oncology, version 2.2018, NCCN clinical practice guidelines in oncology. J Natl Compr Canc Netw. 2018;16(1):66-97.

8. Albritton KH, Coccia P. Influencing referral of adolescents and young adults with cancer to sites with higher rates of trial enrollment. Pediatrics. 2014;133(3 Suppl):S104-8.

9. Coccia PF, Altman J, Bhatia S, et al. Adolescent and young adult oncology. Clinical practice guidelines in oncology. J Natl Compr Canc Netw. 2012;10(9):1112-50.

10. Robbins AS, Lerro CC, Barr RD. Insurance status and distantstage disease at diagnosis among adolescent and young adult patients with cancer aged 15 to 39 years: National Cancer Data Base, 2004 through 2010. Cancer. 2014;120(8):1212-9.

11. Colton MD, Goulding D, Beltrami A, et al. A U.S. population-based study of insurance disparities in cancer survival among adolescents and young adults. Cancer Med. 2019;8(10):4867-74.

12. Greenberg M, Klassen A, Gafni A, et al. Outcomes and metrics: measuring the impact of a comprehensive adolescent and young adult cancer program. Cancer. 2011;117(10 Suppl): 2342-50.

13. Ferrari A, Thomas D, Franklin AR, et al. Starting an adolescent and young adult program: some success stories and some obstacles to overcome. J Clin Oncol. 2010;28(32):4850-7.

14. Crosswell HE, Bomar KN, Vickery N, et al. Trials and tribulations for adolescents and young adults with cancer: measuring the impact of a community-based program. J Natl Compr Canc Netw. 2017;15(9):1171-6.

15. Siegel RD. ASCO Quality Care Symposium 2016 Exemplars in Quality and Identifying High-Quality Practice: Delivering Quality in the Community Setting (Bon Secours St Francis Cancer Center). J Oncol Pract. 2016;12(10):898-901.

16. Wharam JF, Ross-Degnan D, Rosenthal MB. The ACA and high-deductible insurance-strategies for sharpening a blunt instrument. N Engl J Med. 2013;369(16):1481-4.
17. Kantarjian H, Steensma D, Rius Sanjuan J, et al. High cancer drug prices in the United States: reasons and proposed solutions. J Oncol Pract. 2014;10(4):e208-11.

18. Siegel RD, Slough RG, Crosswell HE, et al. Drug recovery and Copay Assistance Program in a Community Cancer Center: charity and challenges. J Oncol Pract. 2019;15(7): e628-35.

19. Conley AP, Trinh VA, Zobniw CM, et al. Positive tumor response to combined checkpoint inhibitors in a patient with refractory alveolar soft part sarcoma: a case report. J Glob Oncol. 2018;4:1-6.

20. D'Angelo SP, Mahoney MR, Van Tine BA, et al. Nivolumab with or without ipilimumab treatment for metastatic sarcoma (Alliance A091401): two open-label, non-comparative, randomised, phase 2 trials. Lancet Oncol. 2018;19(3):416-26.

21. Portera CA, Jr., Ho V, Patel SR, et al. Alveolar soft part sarcoma: clinical course and patterns of metastasis in 70 patients treated at a single institution. Cancer. 2001;91(3): 585-91.

22. Kummar S, Allen D, Monks A, et al. Cediranib for metastatic alveolar soft part sarcoma. J Clin Oncol. 2013;31(18): 2296-302.

23. Kasper B, Sleijfer S, Litiere S, et al. Long-term responders and survivors on pazopanib for advanced soft tissue sarcomas: subanalysis of two European Organisation for Research and Treatment of Cancer (EORTC) clinical trials 62043 and 62072. Ann Oncol. 2014;25(3):719-24.

24. Kim JR, Moon YJ, Kwon KS, et al. Tumor infiltrating PD1positive lymphocytes and the expression of PD-L1 predict poor prognosis of soft tissue sarcomas. PLoS One. 2013; 8(12):e82870.

25. Paydas S, Bagir EK, Deveci MA, Gonlusen G. Clinical and prognostic significance of PD-1 and PD-L1 expression in sarcomas. Med Oncol. 2016;33(8):93.

26. Tawbi HA, Burgess M, Bolejack V, et al. Pembrolizumab in advanced soft-tissue sarcoma and bone sarcoma (SARC028): a multicentre, two-cohort, single-arm, open-label, phase 2 trial. Lancet Oncol. 2017;18(11):1493-501.

27. O'Sullivan Coyne G SE, Moore N, et al. Phase 2 study of atezolizumab in patients with alveolar soft part sarcoma. Rome, Italy: Connective Tissue Oncology Society, November 14-17, 2018.

Address correspondence to: Howland E. Crosswell, MD

Bon Secours St. Francis Health System St. Francis Cancer Center 104 Innovation Drive Greenville, SC 29601-3207 USA

Email: hal_crosswell@bshsi.org 